\title{
Silver as Seed-Particle Material for GaAs Nanowires-Dictating Crystal Phase and Growth Direction by Substrate Orientation
}

Lindberg, Anna Helmi Caroline; Whiticar, Alexander; Dick, Kimberly A.; Skold, Niklas; Nygård, Jesper; Bolinsson, Jessica

\section{Published in:}

Nano Letters

DOI:

10.1021/acs.nanolett.5b04218

Publication date:

2016

Document version

Publisher's PDF, also known as Version of record

Document license:

Other

Citation for published version (APA):

Lindberg, A. H. C., Whiticar, A., Dick, K. A., Skold, N., Nygård, J., \& Bolinsson, J. (2016). Silver as Seed-Particle Material for GaAs Nanowires-Dictating Crystal Phase and Growth Direction by Substrate Orientation. Nano Letters, 16(4), 2181-2188. https://doi.org/10.1021/acs.nanolett.5b04218 


\title{
Silver as Seed-Particle Material for GaAs Nanowires-Dictating Crystal Phase and Growth Direction by Substrate Orientation
}

\author{
Caroline Lindberg, ${ }^{\dagger}$ Alexander Whiticar, ${ }^{\dagger}$ Kimberly A. Dick, ${ }^{\ddagger}$, Niklas Sköld, $^{\ddagger}$ Jesper Nygård, \\ and Jessica Bolinsson* ${ }^{*} \dagger$ \\ ${ }^{\dagger}$ Center for Quantum Devices \& Nano-Science Center, Niels Bohr Institute, University of Copenhagen, Nørregade 10,1165 \\ København, Denmark \\ ${ }^{\ddagger}$ Solid State Physics/NanoLund, ${ }^{\S}$ Center for Analysis and Synthesis, Lund University, 22100 Lund, Sweden
}

\section{Supporting Information}

ABSTRACT: Here we investigate the feasibility of silver as seed-particle material to synthesize GaAs nanowires and show that both crystal phase and growth direction can be controlled by choice of substrate orientation. A (111)B substrate orientation can be used to form vertically aligned wurtzite GaAs nanowires and a (100) substrate orientation to form vertically aligned zinc blende GaAs nanowires. A 45$50 \%$ yield of vertical nanowire growth is achieved on the (100) substrate orientation without employing any type of surface modification or nucleation strategy to promote a vertical growth direction. In addition, photoluminescence measurements reveal that the photon emission from the silver seeded wurtzite GaAs nanowires is characterized by a single

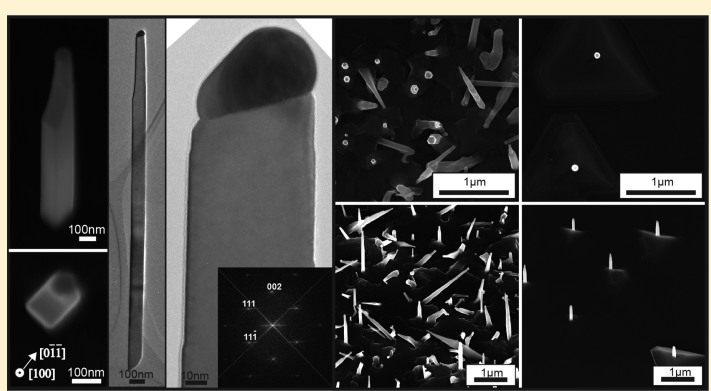
and narrow emission peak at $1.52 \mathrm{eV}$.

KEYWORDS: GaAs, nanowire, silver catalyst, III-V semiconductors, growth direction, photoluminescence

$\mathrm{T}$ he research topic of III-V semiconductor nanowires (NWs) has expanded enormously during the last two decades. Many proof-of-principle applications and devices have been realized, which encompasses several commercially important components and devices as for example transistors, ${ }^{1}$ lasers, ${ }^{2}$ light-emitting diodes, ${ }^{3}$ and solar cells. ${ }^{4,5}$ Recent applications of III-V NWs include advanced concepts within physics such as experiments on the detection of a new type of quasi-particles, the Majorana Fermions, using hybrid superconductor-semiconductor NW devices ${ }^{6}$ and realizing quantum bits in a NW using spin-orbit interactions. ${ }^{7}$ In this Letter we explore a new method to fabricate GaAs NWs, a subgroup of the III-V NWs which has shown great promise for many important applications in optoelectronics. ${ }^{3,8-10}$ Recent advancement include highly efficient solar cells based on GaAs NW arrays $^{11}$ and a near-infrared NW plasmon laser based on GaAs-AlGaAs core-shell NWs. ${ }^{12}$

III-V NWs (including GaAs NWs) are most often grown epitaxially by a bottom-up approach using gold ( $\mathrm{Au}$ ) nanoparticles to seed or catalyze one-dimensional crystalline growth. ${ }^{13}$ However, exploring Au-free methods to synthesize NWs is of significant importance for future applications of NWs since the $\mathrm{Au}$ itself may impair the possibility for NW-based devices to be successfully integrated with Si electronics. ${ }^{14,15}$ One example of an Au-free method is using a self-seeding strategy in which the group III component of the NW plays the role of seed-particle/seed-droplet. ${ }^{16-18}$ Many impressive reports involve this so-called self-catalyzed growth, and this approach has for example been used to successfully grow GaAs
NWs on Si wafers. ${ }^{19-24}$ A few reports include the use of foreign seed metals other than $\mathrm{Au}$ for III-V NW growth. ${ }^{15}$ Investigations involving growth of $\mathrm{GaAs} \mathrm{NWs}$ using $\mathrm{Ni}$, Fe, $\mathrm{Mn}, \mathrm{Pd}$, and $\mathrm{Sn}^{15,25,26}$ have been reported. However, most III$\mathrm{V}$ NW research has been focused on Au seeding and selfseeding so far. We believe that it is highly motivated to put an increased research focus on other seed-particle materials for the fabrication of NWs. It can be expected that new insights will come from investigating this largely unexplored parameter, and it may lead to new approaches to overcome some of the challenges and difficulties that still remain in the pursue of perfect and tunable heterostructured NWs, crystal structure purity, and accessible NW growth directions. For example, an interesting approach to improve the abruptness of axial interfaces in $\mathrm{Si} / \mathrm{Ge}$ heterostructured $\mathrm{NWs}$ was recently presented by Chou et $\mathrm{al}^{27}$ using an $\mathrm{Ag}-\mathrm{Au}$ alloy. In their work they show that, by turning to this alloy as seed material instead of using Au solely, it is possible to tailor the state of the catalyst during growth and reduce the so-called reservoir effect. $^{28}$ One may anticipate that the reservoir effect should be affected by choice of seed material also for other material systems since both eutectic temperatures and solubilities are material dependent. In addition, some material combinations are not completely accessible for NW heterostructures as the

Received: October 16, 2015

Revised: February 29, 2016

Published: March 21, 2016 
change of material along the length of the NWs may lead to kinking for some types of axial heterostructures. ${ }^{29,30}$ For example, although switching from InAs to GaAs is possible while still retaining a straight NW morphology the opposite switching, going abruptly from GaAs to InAs, has been found to induce a change of growth direction (i.e., so-called kinking) for $\mathrm{Au}$ seeded NWs. ${ }^{29}$ Recently it has been shown that through extensive tuning of growth parameters, including careful control of crystal phases and using growth interruptions, it is possible to keep a straight $\mathrm{NW}$ morphology for this particular material combination. ${ }^{31}$ However, in order to unlock the full potential of bandgap engineering in III-V NWs, the challenge of keeping a straight morphology for axial heterostructured NWs remains to be solved for several material combinations.

III-V NWs are known to exhibit so-called polytypism, and much efforts have been devoted to understand and control crystal phase purity. ${ }^{32-36}$ The tendency for polytypism in III-V NWs has opened up for new types of applications. For example, crystal-phase quantum dots in NWs have been reported. ${ }^{37-39}$ Engineering the crystal phases in III-V NWs and reaching high crystal phase purity through careful tuning of growth parameters is achievable in most cases, and recent reports on GaAs NWs show impressive crystal phase purity. ${ }^{24,40-43}$ However, it is challenging and realizing $100 \%$ yield of NWs with a single crystal phase across the full sample area is not in any way straightforward. In addition, many of the current approaches rely on careful tuning of growth parameters which also affects the interplay between radial and axial growth rates (i.e., may cause unintentional radial overgrowth for example) and impurity incorporation. ${ }^{44}$ Polytypism in III-V NWs is related to the fact that they generally prefer to grow in the $<111>\mathrm{B}$ direction. Work has also been done to investigate the possibility to grow the NWs in other directions as an approach to improve their crystal quality ${ }^{45}$ and also because $\{111\}$ surface orientations are not particularly compatible with device processing used in the semiconductor industry, where $\{001\}$ is much more common. ${ }^{45-47}$ With the exception of a few reports showing an impressive yield through a careful choice of growth parameters and nucleation conditions, ${ }^{48}$ achieving NW growth in other directions than the $\langle 111\rangle \mathrm{B}$ has been shown to be rather challenging. ${ }^{45,47,49}$ At present it is therefore almost a necessity to use $\{111\} \mathrm{B}$ oriented substrates if one wants to grow vertical III-V NWs from III-V wafers. It should be noted that the nonpolar (111) substrate orientation of Si have also been used to successfully grow a high yield of vertical GaAs NWs, see for example refs 19, 20, 22, and 50 and references within. Looking at the literature on particle-seeded NW growth using other seeds than $\mathrm{Au}$, a common denominator appears to be the larger variation in growth direction compared to using Au. ${ }^{15}$ For example, a recent report of Pd catalyzed InAs NWs by $\mathrm{Xu}$ et al. shows InAs NW growth in the $\langle\overline{11} 0\rangle$ directions from a (111)B oriented GaAs substrate surface. ${ }^{51}$ To summarize, exploring other seed materials could reveal many new and important possibilities for further engineering of III-V NWs, and reports in the literature are encouraging considering axial heterostructured NWs, crystal phase engineering, as well as accessible NW growth directions.

In this work we explore the feasibility of using silver $(\mathrm{Ag})$ as seed-particle material by investigating growth of GaAs NWs by molecular beam epitaxy (MBE). This is, according to the best of our knowledge, the first report on Ag seeded GaAs NWs. Recent reports suggest that $\mathrm{Ag}$ nanoparticles can be used to nucleate and grow NWs of InP, ${ }^{52} \mathrm{InSb},{ }^{53} \mathrm{InAs},{ }^{54}$ and GaP. ${ }^{55}$
Although these reports show varied yield and control of important NW properties (here meaning crystal phase and growth direction), they do suggest $\mathrm{Ag}$ as an interesting candidate for particle-seeded synthesis of III-V NWs in general for all of the commonly used epitaxy systems (i.e., metal-organic chemical vapor deposition/metal-organic vapor phase epitaxy, chemical beam epitaxy, and MBE). We find that GaAs NWs can indeed be grown using Ag as seed material, potentially offering some important benefits compared to Au. Here we show that $\mathrm{Ag}$ can be used as seed material to form GaAs NWs of a high-quality crystal structure, both zinc blende (ZB) and wurtzite (WZ) phases, and that Ag seeding provides the possibility to select growth direction and crystal phase by simply using different substrate orientation. A (100) substrate orientation can be used to form vertically aligned ZB NWs and a (111)B orientation can be used for vertically aligned WZ NWs. In particular, $45-50 \%$ of the NWs become vertically aligned on the (100) GaAs surface without any particular surface treatment or other efforts to promote a change in growth direction. In addition, photoluminescence (PL) measurements of the $\mathrm{Ag}$ seeded $\mathrm{WZ}$ GaAs NWs reveal a strong and narrow emission peak at $1.52 \mathrm{eV}$, indicating a bright future for these type of NWs in future device applications.

In this study we deposited thin Ag films onto either (111)B or (100) GaAs wafers by electron beam evaporation. Most of the investigations were carried out using the (111)B surface orientation and most of the observations which will be discussed later on concern growths using this orientation. We will state clearly when the result presented concerns the (100) orientation to separate observations concerning the two different surface orientations. To be able to directly observe the effect of Ag, part of the surface area of the wafers was covered during the metal deposition to define Ag-free areas on each sample. The film is transformed into nanoparticles through thin film annealing, ${ }^{56}$ and the growth was carried out using a Varian Gen II solid source MBE system. After establishing an annealing procedure for deoxidation and the formation of $\mathrm{Ag}$ nanoparticles (see section I in Supporting Information), we carried out various growths to explore the possibility of using $\mathrm{Ag}$ particles as seeds for growth of GaAs NWs. In particular, a temperature series for fixed As and $\mathrm{Ga}$ fluxes was done in a temperature range of $400-600{ }^{\circ} \mathrm{C}$, in steps of $50{ }^{\circ} \mathrm{C}$, using GaAs (111)B. Additional growths were also carried out in the temperature range between 550 and $600{ }^{\circ} \mathrm{C}$ to further investigate certain changes in NW density and NW growth direction that was found to occur in this temperature interval. For comparison, $\mathrm{Au}$ seeded GaAs NWs were also grown from (111)B GaAs wafers (see section VI in Supporting Information). For experimental details concerning the growth process, see section I in the Supporting Information.

The NW samples were characterized by scanning electron microscopy (SEM) using a Raith eLine 100 system and a JEOL JSM-6320F SEM. This characterization involved NW morphology, density, vertical yield, length, and diameter (see also sections II and III in Supporting Information). The crystal structures of the NWs were determined by transmission electron microscopy (TEM): a JEOL 3000F operating at 300 $\mathrm{kV}$ and a Philips CM20 operating at $200 \mathrm{kV}$. By moving $\mathrm{Cu}$ grids with lacey carbon film on a small part of the wafer, NWs were physically transferred to the grids. The chemical composition of the particles and the NWs were analyzed using electron dispersive spectroscopy (EDS) with the JEOL $3000 \mathrm{~F}$ in scanning TEM-high angle annular dark field 
$400^{\circ} \mathrm{C}$
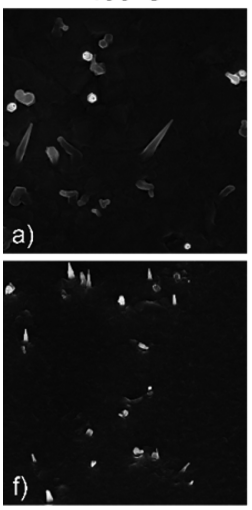

$450^{\circ} \mathrm{C}$
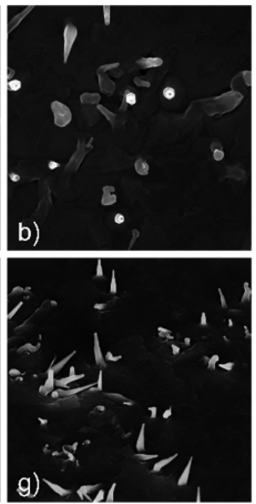

$500^{\circ} \mathrm{C}$

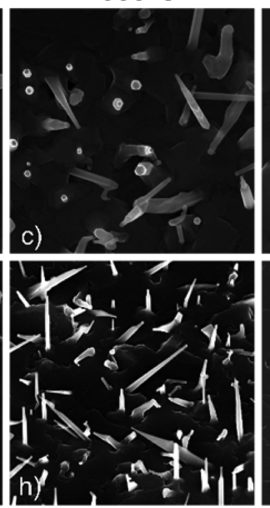

$550^{\circ} \mathrm{C}$
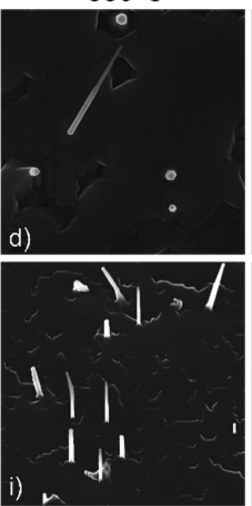

$600^{\circ} \mathrm{C}$

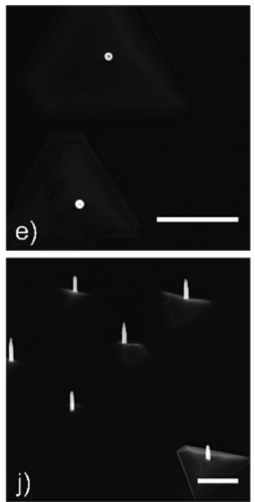

Figure 1. SEM images illustrating the influence of temperature on the Ag seeded growth of GaAs NWs grown on GaAs (111)B. The SEM images in the top row are top-view images, while the lower row is tilted view $\left(45^{\circ}\right)$ of the same sample (i.e., a and $\mathrm{f}$ are from the sample grown at $400{ }^{\circ} \mathrm{C}$, $\mathrm{b}$ and $\mathrm{g}$ from the sample grown at $450^{\circ} \mathrm{C}$ and so on). Scale bars are $1 \mu \mathrm{m}$.

(HAADF) mode. Photoluminescence measurements (PL) were performed on single NWs after these had been broken off from the substrate and transferred to a gold surface. The measurements were performed with the samples cooled to $<10 \mathrm{~K}$ using a continuous wave laser and a wavelength of $532 \mathrm{~nm}$ for excitation. The light was focused onto the sample using a microscope objective lens, which was also used to collect the emission from the NWs. The collected emission was then dispersed by a grating spectrometer onto a charge coupled device.

Figure 1 shows SEM images of a series of samples grown on GaAs (111)B substrates using the same Ga and As fluxes but different growth temperatures. At all temperatures, although it was varied over a total range of $200{ }^{\circ} \mathrm{C}$, GaAs NWs form. At the highest temperature in our study, $600{ }^{\circ} \mathrm{C}$, we observe that all NWs are vertically aligned to the substrate surface (i.e., apparently grown in the $<111>\mathrm{B}$ direction). For growth temperatures below $550{ }^{\circ} \mathrm{C}$, the $\mathrm{Ag}$ seeded growth is unstable, and the NWs are synthesized in various directions and shapes, showing curly NW-like objects, kinked NWs, and NWs formed in several different inclined directions to the substrate surface. The ratio between vertical NWs versus nonvertical NWs for the different growth temperatures are presented in Figure S1 in the Supporting Information.

The total density of NWs varies significantly with growth temperature. At the highest temperature, we find a total NW density of 0.02 per $\mu \mathrm{m}^{2}$, while the total NW density at the lowest temperature is 0.85 per $\mu \mathrm{m}^{2}$ (these data are displayed in Figure S1 in Supporting Information). Since we "shadow" part of the substrate surface area during the thin film deposition of the Ag (as described above), there are also Ag-free surface areas on all our samples. For all samples, these areas were carefully investigated post-growth by SEM to compare with observations made on the Ag-covered parts. For all samples seen in Figure 1, we did not observe any signs of NW growth present on the Agfree areas, and it is thus possible to conclude that the $\mathrm{Ag}$ is indeed enabling the NW growth on these samples. Further, energy dispersive X-ray spectroscopy (EDS) measurements done in conjunction with TEM analysis confirmed that the particle at the tip of the NWs is $\sim 99$ at. \% Ag (EDS data is presented in section $\mathrm{V}$ in Supporting Information).

Extensive SEM imaging was carried out on many samples to investigate if there are nanoparticles on the tips of the NWs and what spread we could find in particle diameter for $\mathrm{Ag}$ seeded
GaAs NWs. We find that Ag seeding of GaAs NWs appear to, at least, be possible for nanoparticles in sizes from around 10 $\mathrm{nm}$ to above $150 \mathrm{~nm}$ in the temperature range we investigated here (i.e., $400-600{ }^{\circ} \mathrm{C}$ ). The spread in particle diameter can also be seen in Figure S2 in section III in Supporting Information. We note that, at lower growth temperatures, there is a more pronounced tapering along the entire NWs, while at higher temperatures the shape of the NWs is more homogeneous except for the top of the NW where it is found to taper off toward the NW tip. SEM images of the overall NW morphology for growth temperatures of 400 and $600{ }^{\circ} \mathrm{C}$ are included in Supporting Information; see section IV.

As discussed above, the vertical yield of the GaAs NWs varies strongly with growth temperature, and we therefore carried out several growths in the temperature range of around 580-600 ${ }^{\circ} \mathrm{C}$ to explore whether the results are reproducible and if a high yield of vertical NWs is also possible when going slightly below $600{ }^{\circ} \mathrm{C}$. We find that our results are indeed reproducible, and the temperature window to form the GaAs NWs using Ag seeds appears to allow for a temperature variation from growth to growth in the order of at least $10-20{ }^{\circ} \mathrm{C}$. After establishing the temperature dependence of nonvertical versus vertical NWs using SEM characterization as outlined above, we continued our investigations by more detailed characterization of the NWs by TEM. For these investigations we focused on studying NWs grown under conditions at which nonvertical growth is avoided, i.e., for a growth temperature of around $580-600{ }^{\circ} \mathrm{C}$. In Figure 2 , we show TEM images of a typical NW grown using such conditions. The TEM measurements confirm that the NWs are grown in a $<111>$ direction (presumably $<111>\mathrm{B}$ due to their alignment with the substrate, but the actual polarity of the growth direction have not been verified). In general, the NWs have an almost pure WZ crystal structure with only a few defects in the NW region closest to the particle. A change in the crystal structure close to the tip of NWs has been reported earlier and is generally interpreted as an effect of particle-seeded growth occurring during the cooling process (i.e., a so-called cooling neck). ${ }^{57}$

Moreover, by varying the growth time (30 or $60 \mathrm{~min}$ ), we note that the length of the NWs scaled directly with growth time (see section III in Supporting Information). In addition, for samples grown for $60 \mathrm{~min}$, we made a few observations of NWs which did not appear to have a particle on top (see also Figure S5 in section V in Supporting Information). Further 


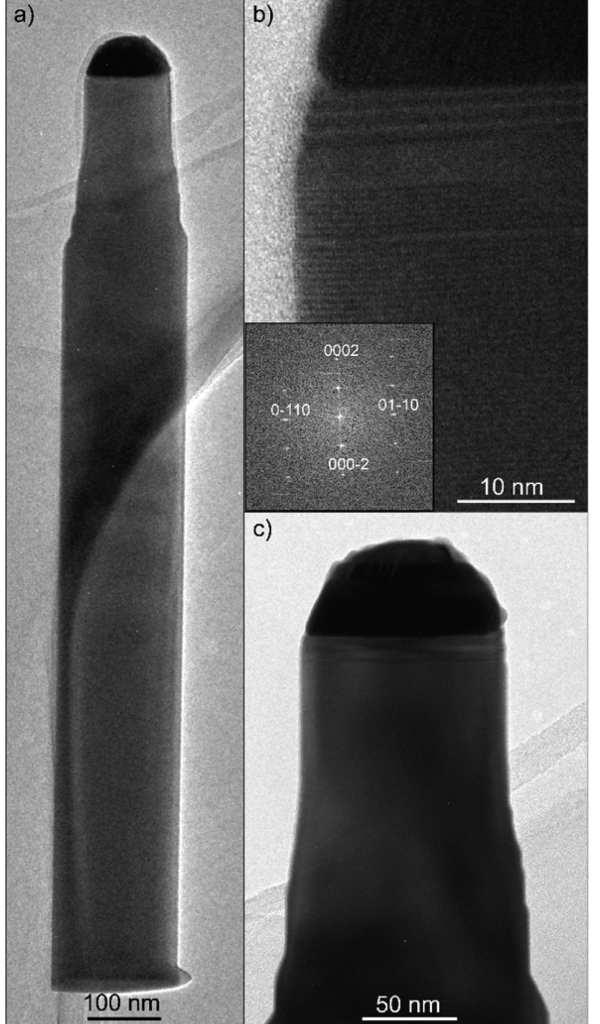

d)

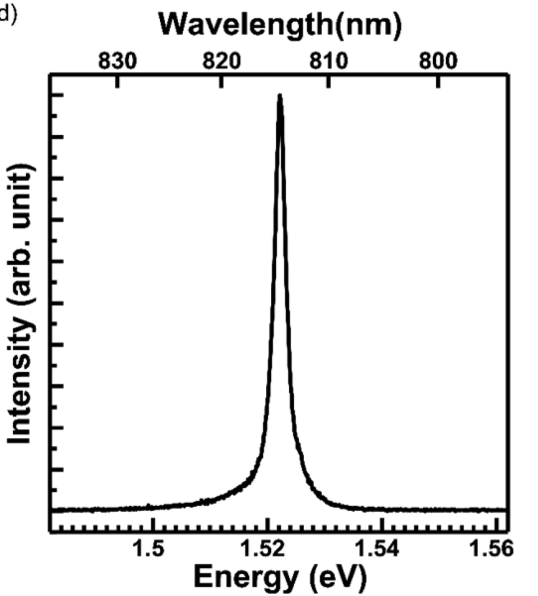

Figure 2. Parts a $-\mathrm{c}$ show TEM images in a $<110>$ viewing direction of a typical Ag seeded GaAs NW grown at $600{ }^{\circ} \mathrm{C}$ and using an (111)B substrate orientation. As is seen, the $\mathrm{NW}$ has an excellent WZ crystal structure, with only a few defects near the interface to the seedparticle. Inset in b shows a FFT confirming that the crystal structure is WZ. Part $d$ is a PL emission spectrum of the same type of NW as the one shown in $a-c$.

TEM characterization of NWs from such samples confirmed a particular type of NWs which has a faceted tip without a particle, and no measurable signal from $\mathrm{Ag}$ is found neither at the tip region nor along the length of the these NWs in our EDS measurements. At this point, our hypothesis is that these still have started off as Ag seeded NWs but then at some point the particle has become unstable, perhaps due to a reduced supply of Ga to the seed-particle as the distance to the substrate surface increases, causing the Ag to "escape" and thus interrupting the Ag particle-seeded NW growth. This interpretation is supported by other reports of this phenomenon $^{58}$ and an apparent correlation between the presence of this NW type and an increased growth time (i.e., increased NW length).

The results presented above clearly demonstrate the feasibility of using Ag seeding for the fabrication of GaAs NWs with an excellent crystal quality. Since GaAs NWs are most interesting for optical applications, it is of importance to investigate their optical properties. Photoluminescence (PL) measurements were carried out on single NWs with the same crystal quality as shown for the NW seen in Figure $2 \mathrm{a}-\mathrm{c}$, i.e., pure WZ crystal structure. A representative emission spectrum from these measurements (measured at $5 \mathrm{~K}$ ) is included in Figure 2d). The photon emission from the $\mathrm{Ag}$ seeded $\mathrm{WZ}$ GaAs NWs is characterized by a single and narrow emission peak centered at $1.520 \pm 0.002 \mathrm{eV}$ (fwhm of $5 \pm 2 \mathrm{meV}$ ), i.e., at an energy which is commonly associated with the band gap of $\mathrm{ZB} \mathrm{GaAs}$. We note that there is still some debate regarding the exact band gap energy of WZ GaAs and the corresponding exciton emission energy. ${ }^{59,60}$ Since GaAs NWs are often found to have a mixed crystal structure, the type-II band offset between the $\mathrm{ZB}$ and $\mathrm{WZ}$ crystal phases have certainly made it problematic to directly measure the band gap of $\mathrm{WZ}$ GaAs and separate between emission associate with the $\mathrm{WZ}$ phase and twin defects. ${ }^{44,60,61}$ Our observation of photon emission from extremely pure WZ GaAs NWs at the same energy as the band gap of $\mathrm{ZB} G \mathrm{GaAs}$ is in agreement with recent reports of $\mathrm{Au}$ seeded WZ GaAs NWs. ${ }^{59,62}$ Further, no discernible peak other than the bandgap emission was found. Ag defects in GaAs are expected to show a PL peak in the range 1.2-1.28 eV (caused by a deep level $0.238 \mathrm{eV}$ above the valence band edge). ${ }^{63,64}$ No such peak was found in this study, indicating minimal or no incorporation of $\mathrm{Ag}$ during growth. We believe that the position of the emission peak, and the fact that its width is comparable to the most narrow ones that have been reported so far, ${ }^{59,62}$ is strongly in favor for further research of using Ag seeding for the fabrication of GaAs NWs for optoelectronic device applications. Based on our observations, it does not seem to be a problem with unintentional $\mathrm{Ag}$ doping or nonradiative recombination due to $\mathrm{Ag}$ impurities in the NWs. It is worth mentioning that compared to $\mathrm{Au}, \mathrm{Ag}$ has a diffusion coefficient in Si which is 2 orders of magnitude lower, in favor for Ag seeding compared to $\mathrm{Au}$ looking at future integration with $\mathrm{Si}^{.85} \mathrm{Ag}$ is in fact already used in the mass production of solar cells based on $\mathrm{Si}^{66}$ and could possibly be more acceptable in the $\mathrm{Si}$ processing compared to Au.

For the completeness of this work, we also make some comparison between Ag seeded NW growth on (111)B GaAs wafers and the more conventional choice of using $\mathrm{Au}$ as seedparticle material. There are many reports on Au seeded GaAs NWs grown by MBE. ${ }^{67-69}$ However, it is difficult to directly translate growth parameters, the temperature in particular, in between different epitaxy machines. We have therefore carried out a few growths of Au seeded GaAs NWs on (111)B GaAs wafers to be able to directly compare this with the growth of $\mathrm{Ag}$ seeded GaAs NWs by the same epitaxy system (experimental details for the $\mathrm{Au}$ seeded $\mathrm{GaAs}$ is given in section VI in Supporting Information). For Au seeded GaAs NWs we note that at higher temperatures $\left(\sim 550{ }^{\circ} \mathrm{C}\right)$ nonvertical $\mathrm{NWs}$ becomes more common (similar morphology and occurrence of nonvertical NWs was also observed by $\mathrm{Li}$ et al. ${ }^{70}$ for a growth temperature of $620^{\circ} \mathrm{C}$ ), while decreasing the temperature gives an increased ratio of vertical NWs. For a growth temperature of $520{ }^{\circ} \mathrm{C}$ we find that in principle all NWs are vertical (see Figure S6 in Supporting Information). A further decrease of the 
growth temperature to $480{ }^{\circ} \mathrm{C}$ resulted in a different $\mathrm{NW}$ morphology (increased tapering), but the yield of vertical ones is the same as when using a growth temperature of $520{ }^{\circ} \mathrm{C}$. Comparing this result with what is observed for $\mathrm{Ag}$ seeded GaAs NW growth, it can be concluded that there is a temperature difference of about $60{ }^{\circ} \mathrm{C}$ between the two types of temperature thresholds with respect to realizing only vertical NW growth: for Au seeding it is required to use growth temperature below $550{ }^{\circ} \mathrm{C}$, around $520{ }^{\circ} \mathrm{C}$, while for $\mathrm{Ag}$ seeding it is required to be above $550{ }^{\circ} \mathrm{C}$, around $580{ }^{\circ} \mathrm{C}$ to avoid nonvertical NWs.

Due to the novelty of this work, it is interesting to consider possible growth mechanism behind Ag seeding. One important aspect is whether the growth proceeds by a so-called vaporliquid-solid mechanism ${ }^{71}$ or some vapor-solid-solid ${ }^{57}$ one, or perhaps both are possible. As is mentioned above, EDS measurements of the seed particles indicated an $\mathrm{Ag}$ concentration of almost 100\% (99 at. \% Ag, see section V in Supporting Information). Turning to what is known about the binary $\mathrm{Ag}-\mathrm{Ga}$ phase diagram, ${ }^{72}$ the solubility of $\mathrm{Ga}$ in solid $\mathrm{Ag}$ is maximum about 18 at. \% in the temperature range of $400-$ $600{ }^{\circ} \mathrm{C}$. The liquidus minimum is pure $\mathrm{Ga}$, and to reach a liquid alloy between the two atom species the Ga concentration needs to be as high as about 29 at. \% or higher (and a temperature of $612{ }^{\circ} \mathrm{C}$ ). Further, the As-Ag binary phase diagram has an eutectic point at $\sim 540{ }^{\circ} \mathrm{C}^{73}$ for an As concentration of $11 \%$. Considering the growth temperatures we include in this work, VLS growth using an $\mathrm{Ag}-\mathrm{As}$ alloy would require an As concentration of around $11 \%$ or higher, and this type of NW growth would only be accessible in part of the explored temperature range (i.e., above $540{ }^{\circ} \mathrm{C}$ ). There appears to be a distinct change in total density of NWs as well as in the yield of vertical NWs around $550{ }^{\circ} \mathrm{C}$ (Supporting Information, Figure $\mathrm{S} 1$ ). One may speculate that this change is somehow related to the eutectic point reported around this temperature for the $\mathrm{Ag}$-As alloy. Ag-seeded $\mathrm{GaP} \mathrm{NWs}$ have been grown at a temperature of $480{ }^{\circ} \mathrm{C}$ by Huang et al. with a postgrowth particle composition of around $50 \% \mathrm{Ga}, 25 \% \mathrm{Ag}$, and $20 \% \mathrm{P}^{55}$ The authors do not specifically discuss a possible growth mechanism, but from their data and the $\mathrm{Ag}-\mathrm{Ga}$ phase diagram, one may argue that at this high Ga concentration it is possible that the growth actually occurred from a VLS mechanism. Although their experiments differ from the ones presented here (they use chemical beam epitaxy and grew the NWs from Si wafers), it is interesting to compare the particle composition and note that there is a significant difference in NW growth directions. Further, Huang et al. included both $\{111\}$ and $\{001\}$ surface orientations and observe NW growth mainly in the [111] and [110] directions on both surfaces and only NWs with small diameters (less than $20 \mathrm{~nm}$ ). ${ }^{55}$ Comparing with other III-V NWs, we further note that Pan et al. ${ }^{54}$ report InAs NWs using Ag and found a postgrowth composition of around $13 \% \mathrm{In}$ and $86 \% \mathrm{Ag}$ and Vogel et al. ${ }^{53}$ report of InSb NW growth from a "In-rich droplet" and a $14 \%$ concentration of Ag. At this point, we cannot conclude if the Ag seeded GaAs NWs grown here proceed by VLS or VSS or some other type of mechanism that is yet to be described. However, from our results and the literature outlined above, it is strongly suggested that Ag seeding can be used to synthesis NWs both for a solid and a liquid Ag. In addition, any theoretical modeling involving Ag seeding of III-V NWs needs to take into account both possible alloying with the group III and the group V species.
To conclude this work, we also explore the possibility for $\mathrm{Ag}$ seeded NW growth using another surface orientation, the (100), and the observations discussed below concerns this particular surface. As outlined earlier, it is not known what effect the seed-particle material has on the growth direction of the NWs. Here we therefore included growths where we apply the same growth condition as was found to be successful for NW growth on GaAs (111)B to NW growth on GaAs (100). We do not apply any particular surface treatment which is commonly used in order to modify the substrate surface and promote growth in other directions than $<111>\mathrm{B}^{45,46,48,74,75}$ In Figure 3, we show SEM images of a sample where Ag seeded

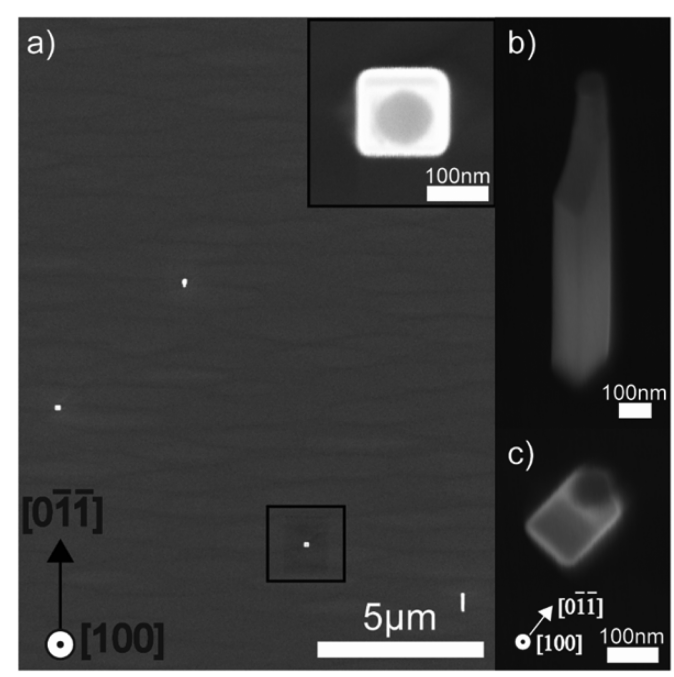

Figure 3. SEM images of GaAs NWs grown on GaAs (100). (a) Topview showing an overview and the inset in upper right corner shows a higher magnification image of one of the [100] grown NWs. Note the square shaped cross-section of the NW and the $\{110\}$-type facets. Parts $b$ and $c$ show higher magnification SEM images of a NW in $15^{\circ}$ tilt and top-view, respectively.

GaAs NW growth was carried out on GaAs (100). As is seen from the overview image shown in a, the overall NW density is low (the low NW density of the (100) surface will be discussed below). The yield of vertically aligned NWs versus nonvertically aligned NWs is, however, surprisingly high and very well represented by this particular image. SEM analysis showed that around $45-50 \%$ of the NWs grown on the 100 substrate are vertically aligned, thus indicating that a large fraction of the NWs grew in the [100] growth direction. According to the authors' knowledge, this report represents the highest yield of vertical NWs on $\{100\}$ type surfaces without surface treatment or other to promote the vertical yield. ${ }^{47-49}$ TEM characterization confirmed that the growth direction is $\langle 100\rangle$ and that the NWs are pure ZB as one would expect for NWs grown in this direction; ${ }^{49,74}$ see Figure 4. Upon closer inspection, it is observed that the interface plane between the GaAs NW and the Ag seed-particle appears to be nonperpendicular to the $<100>$ growth direction of the NW. As seen from Figure 4d), this particular NW has an interface surface to its seed-particle tilted about $14^{\circ}$ from the (100) plane. We note that possible planes close to such an angle are (115) and (116) corresponding to angles of $15.8^{\circ}$ and $13.3^{\circ}$, respectively. Further on, EDS measurements suggest that the composition of the particle is the same for the $<100\rangle$ grown NWs as the NWs grown from (111)B substrates; almost purely $\mathrm{Ag}$ ( $\sim 99$ at. \% 


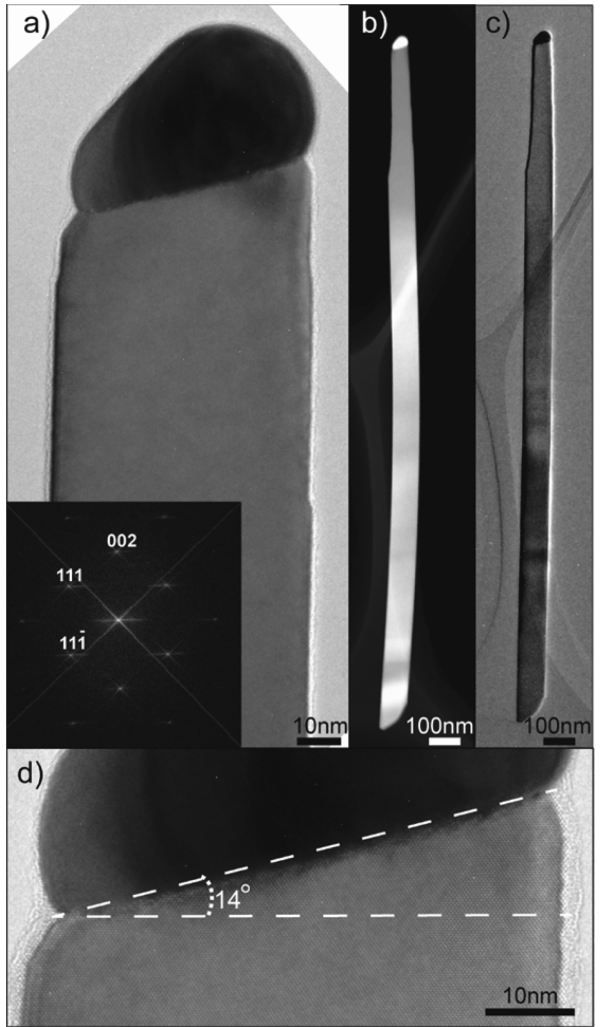

Figure 4. TEM images in a $<110>$ viewing direction of an $\mathrm{Ag}$ seeded GaAs NW grown on GaAs (100). Inset in a is the FFT, confirming the crystal structure ZB. The interface between the GaAs material and Ag particle is tilted by about $14^{\circ}$ from the (100) plane, and the NW is free of crystal defects.

Ag, see Supporting Information, Figure S4). Perhaps most intriguing, an identical particle composition during growth of the NWs from the (100) and the (111)B orientation suggests that it is the substrate surface orientation that directly dictates whether the NWs become $\mathrm{WZ}$ or $\mathrm{ZB}$ and whether they grow in a $<111>$ or a $<100>$ direction.

As mentioned above, the overall NW density on the (100) surface is low. It should be noted, however, that we have carried out several growths using these conditions to confirm that this is indeed reproducible and that the area shown in Figure $3 \mathrm{a}$ is representative for this type of samples. We aim to continue with tuning of the growth parameters, possibly also exploring if the annealing step should be modified, to increase the total yield of nucleated NWs on the (100) surface and to investigate if the vertical yield can be improved further. A few growths have been done in which the growth conditions were slightly modified. From these we observe that the overall yield of NWs is influenced by the Ga flux, where a higher flux can be used to improve the overall density of NWs. In particular, we note that the NW growth on the (100) surface appear to be much more sensitive to changes in the Ga flux compared to the (111)B. A decrease in Ga flux (in the order of 15\%) can result in going from having a low density of NWs to having no NWs at all on the (100) surface. The same trend is observed when the temperature is increased. While vertical NW growth from the (111)B substrate orientation is stable in the temperature range of $580-600{ }^{\circ} \mathrm{C}$, there are no NWs to be found on the (100) surface when the growth temperature is set to $600{ }^{\circ} \mathrm{C}$. For growth from the (100) surface it is necessary to be slightly below $600{ }^{\circ} \mathrm{C}$ (around $580{ }^{\circ} \mathrm{C}$ ) to achieve the type of $\mathrm{NW}$ growth as is illustrated in Figure 3. At present, we can only speculate that the NW nucleation is more problematic on the (100) oriented surface compared to the (111)B for the growth conditions we used in this work and conclude that further efforts are needed to increase the density of NWs on the (100) surface while keeping a good yield of vertical ones. Possibly, nucleation problems and a competing overgrowth of GaAs material on the substrate surface (which also will be different on the (111)B and the (100)) is part of the explanation for the sparse density of NWs on the (100) surface that we observe here.

In summary, we have demonstrated the feasibility of using $\mathrm{Ag}$ as seed material for particle-seeded growth of GaAs NWs and shown that GaAs NWs grown from Ag seed-particles are promising for future applications relying on well-defined photon emission. Both high quality WZ and ZB NWs can be grown vertically using the same growth conditions and be controlled by the choice of substrate surface orientation, where a (111)B surface gives WZ NWs and a (100) surface gives ZB NWs. The WZ GaAs NWs show a surprisingly strong and narrow emission at $1.52 \mathrm{eV}$, with a fwhm of only around $5 \mathrm{meV}$. For the (111)B surface a vertical yield of $100 \%$ have been achieved, while for a (100) surface orientation, about 45-50\% of the NWs are found to be vertically aligned without applying any particular surface treatment or other to promote a change in NW growth direction. We believe that our results encourage further research efforts on $\mathrm{Ag}$ as seed-material for the synthesis of III-V NWs in general. We also hope that our report will open up for engineering of III-V NW properties using various seed materials and that by being able to choose between various nanoparticles will allow for new functionalities of future NWbased devices. Going toward particle-seeded NW growth using other materials than Au could provide a new direction for NWbased devices by making it possible to investigate if the intrinsic properties of different nanoparticles themselves (such as for example their surface plasmon resonance, toxicity, or chemical catalytic effect) could be utilized to improve, or even control, the functionality of the complete NW device.

\section{ASSOCIATED CONTENT}

\section{S Supporting Information}

The Supporting Information is available free of charge on the ACS Publications website at DOI: 10.1021/acs.nanolett.5b04218.

Experimental details on growth process, data of nanowire density and vertical yield as a function of growth temperature, nanowire growth rate as a function of particle diameter, nanowire morphology, EDX analysis of seed-particle composition, and SEM images of Au seeded GaAs nanowires (PDF)

\section{AUTHOR INFORMATION}

\section{Corresponding Author}

*E-mail: jessica.bolinsson@nbi.ku.dk.

\section{Notes}

The authors declare no competing financial interest.

\section{ACKNOWLEDGMENTS}

C.L., J.N., and J.B. acknowledge financial support from the Danish Agency for Science Technology and Innovation (Council for Strategic Research-ANaCell project) and the University of Copenhagen program on Synthetic Biology. J.B. 
also acknowledges financial support from the Villum Foundation (Young Investigator Program, no. 10121). K.A.D. acknowledges financial support from the European Research Council under the European Uniońs Seventh Framework Programme (FP/2007-2013)/ERC Grant Agreement No. 336126 and the Knut and Alice Wallenberg Foundation (KAW), and N.S. acknowledges financial support from the Swedish Research Council (Young Researcher Program, no. 621-2013-5839) and the Crafoord foundation. The Center for Quantum Devices is funded by the Danish National Research Foundation.

\section{REFERENCES}

(1) Riel, H.; Wernersson, L. E.; Hong, M. W.; del Alamo, J. A. MRS Bull. 2014, 39, 668-677.

(2) Couteau, C.; Larrue, A.; Wilhelm, C.; Soci, C. Nanophotonics 2015, 4, 90-107.

(3) Mi, Z.; Chang, Y. L. J. Nanophotonics 2009, 3, 031602.

(4) Kempa, T. J.; Day, R. W.; Kim, S. K.; Park, H. G.; Lieber, C. M. Energy Environ. Sci. 2013, 6, 719-733.

(5) LaPierre, R. R.; Chia, A. C. E.; Gibson, S. J.; Haapamaki, C. M.; Boulanger, J.; Yee, R.; Kuyanov, P.; Zhang, J.; Tajik, N.; Jewell, N.; Rahman, K. M. A. Phys. Status Solidi RRL 2013, 7, 815-830.

(6) Mourik, V.; Zuo, K.; Frolov, S. M.; Plissard, S. R.; Bakkers, E.; Kouwenhoven, L. P. Science 2012, 336, 1003-1007.

(7) Nadj-Perge, S.; Frolov, S. M.; Bakkers, E.; Kouwenhoven, L. P. Nature 2010, 468, 1084-1087.

(8) Joyce, H. J.; Gao, Q.; Hoe Tan, H.; Jagadish, C.; Kim, Y.; Zou, J.; Smith, L. M.; Jackson, H. E.; Yarrison-Rice, J. M.; Parkinson, P.; Johnston, M. B. Prog. Quantum Electron. 2011, 35, 23-75.

(9) Soci, C.; Zhang, A.; Bao, X.-Y.; Kim, H.; Lo, Y.; Wang, D. J. Nanosci. Nanotechnol. 2010, 10, 1430-1449.

(10) Borgström, M. T.; Wallentin, J.; Heurlin, M.; Fält, S.; Wickert, P.; Leene, J.; Magnusson, M. H.; Deppert, K.; Samuelson, L. IEEE J. Sel. Top. Quantum Electron. 2011, 17, 1050-1061.

(11) Åberg, I.; Vescovi, G.; Asoli, D.; Naseem, U.; Gilboy, J. P.; Sundvall, C.; Dahlgren, A.; Svensson, K. E.; Anttu, N.; Björk, M. T.; Samuelson, L. IEEE J. Photovolt. 2016, 6, 185-190.

(12) Ho, J.; Tatebayashi, J.; Sergent, S.; Fong, C. F.; Iwamoto, S.; Arakawa, Y. ACS Photonics 2015, 2, 165-171.

(13) Kolasinski, K. W. Curr. Opin. Solid State Mater. Sci. 2006, 10, $182-191$.

(14) Tavendale, A. J.; Pearton, S. J. J. Phys. C: Solid State Phys. 1983, $16,1665-1673$.

(15) Dick, K. A.; Caroff, P. Nanoscale 2014, 6, 3006-3021.

(16) Fontcuberta i Morral, A.; Colombo, C.; Abstreiter, G.; Arbiol, J.; Morante, J. R. Appl. Phys. Lett. 2008, 92, 063112.

(17) Tersoff, J. Nano Lett. 2015, 15, 6609-6613.

(18) Scarpellini, D.; Somaschini, C.; Fedorov, A.; Bietti, S.; Frigeri, C.; Grillo, V.; Esposito, L.; Salvalaglio, M.; Marzegalli, A.; Montalenti, F.; Bonera, E.; Medaglia, P. G.; Sanguinetti, S. Nano Lett. 2015, 15, 3677-3683.

(19) Dubrovskii, V. G.; Xu, T.; Álvarez, A. D.; Plissard, S. R.; Caroff, P.; Glas, F.; Grandidier, B. Nano Lett. 2015, 15, 5580-5584.

(20) Russo-Averchi, E.; Vukajlovic Plestina, J.; Tütüncüoglu, G.; Matteini, F.; Dalmau-Mallorquí, A.; de la Mata, M.; Rüffer, D.; Potts, H. A.; Arbiol, J.; Conesa-Boj, S.; Fontcuberta i Morral, A. Nano Lett. 2015, 15, 2869-2874.

(21) Munshi, A. M.; Dheeraj, D. L.; Fauske, V. T.; Kim, D. C.; Huh, J.; Reinertsen, J. F.; Ahtapodov, L.; Lee, K. D.; Heidari, B.; van Helvoort, A. T. J.; Fimland, B. O.; Weman, H. Nano Lett. 2014, 14, 960-966.

(22) Plissard, S.; Larrieu, G.; Wallart, X.; Caroff, P. Nanotechnology 2011, 22, 275602.

(23) Cirlin, G. E.; Dubrovskii, V. G.; Samsonenko, Y. B.; Bouravleuv, A. D.; Durose, K.; Proskuryakov, Y. Y.; Mendes, B.; Bowen, L.; Kaliteevski, M. A.; Abram, R. A.; Zeze, D. Phys. Rev. B: Condens. Matter Mater. Phys. 2010, 82, 035302.
(24) Krogstrup, P.; Popovitz-Biro, R; Johnson, E.; Madsen, M. H.; Nygård, J.; Shtrikman, H. Nano Lett. 2010, 10, 4475-4482.

(25) Sun, R.; Jacobsson, D.; Chen, I. J.; Nilsson, M.; Thelander, C.; Lehmann, S.; Dick, K. A. Nano Lett. 2015, 15, 3757-3762.

(26) Hallberg, R. T.; Lehmann, S.; Messing, M. E.; Dick, K. A. J. Mater. Res. 2016, 31, 175-185.

(27) Chou, Y.-C.; Wen, C.-Y.; Reuter, M. C.; Su, D.; Stach, E. A.; Ross, F. M. ACS Nano 2012, 6, 6407-6415.

(28) Li, N.; Tan, T. Y.; Gosele, U. Appl. Phys. A: Mater. Sci. Process. 2008, 90, 591-596.

(29) Dick, K. A.; Kodambaka, S.; Reuter, M. C.; Deppert, K.; Samuelson, L.; Seifert, W.; Wallenberg, L. R.; Ross, F. M. Nano Lett. 2007, 7, 1817-1822.

(30) Paladugu, M.; Zou, J.; Guo, Y. N.; Auchterlonie, G. J.; Joyce, H. J.; Gao, Q.; Tan, H. H.; Jagadish, C.; Kim, Y. Small 2007, 3, 18731877.

(31) Messing, M. E.; Wong-Leung, J.; Zanolli, Z.; Joyce, H. J.; Tan, H. H.; Gao, Q.; Wallenberg, L. R.; Johansson, J.; Jagadish, C. Nano Lett. 2011, 11, 3899-3905.

(32) Caroff, P.; Bolinsson, J.; Johansson, J. IEEE J. Sel. Top. Quantum Electron. 2011, 17, 829-846.

(33) Glas, F.; Harmand, J. C.; Patriarche, G. Phys. Rev. Lett. 2007, 99, 146101.

(34) Dubrovskii, V. G.; Sibirev, N. V. Phys. Rev. B: Condens. Matter Mater. Phys. 2008, 77, 035414.

(35) Johansson, J.; Karlsson, L. S.; Svensson, C. P. T.; Svensson, C.; Mårtensson, T.; Wacaser, B. A.; Deppert, K.; Samuelson, L.; Seifert, W. Nat. Mater. 2006, 5, 574-580.

(36) Joyce, H. J.; Wong-Leung, J.; Gao, Q.; Tan, H. H.; Jagadish, C. Nano Lett. 2010, 10, 908-915.

(37) Akopian, N.; Patriarche, G.; Liu, L.; Harmand, J. C.; Zwiller, V. Nano Lett. 2010, 10, 1198-1201.

(38) Loitsch, B.; Winnerl, J.; Grimaldi, G.; Wierzbowski, J.; Rudolph, D.; Morkötter, S.; Döblinger, M.; Abstreiter, G.; Koblmüller, G.; Finley, J. J. Nano Lett. 2015, 15, 7544-7551.

(39) Vainorius, N.; Lehmann, S.; Jacobsson, D.; Samuelson, L.; Dick, K. A.; Pistol, M.-E. Nano Lett. 2015, 15, 2652-2656.

(40) Joyce, H. J.; Gao, Q.; Tan, H. H.; Jagadish, C.; Kim, Y.; Zhang, X.; Guo, Y.; Zou, J. Nano Lett. 2007, 7, 921-926.

(41) Shtrikman, H.; Popovitz-Biro, R.; Kretinin, A.; Heiblum, M. Nano Lett. 2009, 9, 215-219.

(42) Burgess, T.; Breuer, S.; Caroff, P.; Wong-Leung, J.; Gao, Q.; Hoe Tan, H.; Jagadish, C. ACS Nano 2013, 7, 8105-8114.

(43) Jacobsson, D.; Lehmann, S.; Dick, K. A. Nanoscale 2014, 6, 8257-8264.

(44) Bolinsson, J.; Ek, M.; Trägårdh, J.; Mergenthaler, K.; Jacobsson, D.; Pistol, M. E.; Samuelson, L.; Gustafsson, A. Nano Res. 2014, 7, 473-490.

(45) Fortuna, S. A.; Li, X. L. Semicond. Sci. Technol. 2010, 25, 024005. (46) Wang, J.; Plissard, S.; Hocevar, M.; Vu, T. T. T.; Zehender, T.; Immink, G. G. W.; Verheijen, M. A.; Haverkort, J.; Bakkers, E. Appl. Phys. Lett. 2012, 100, 053107.

(47) Fonseka, H. A.; Caroff, P.; Wong-Leung, J.; Ameruddin, A. S.; Tan, H. H.; Jagadish, C. ACS Nano 2014, 8, 6945-6954.

(48) Wang, J.; Plissard, S. R.; Verheijen, M. A.; Feiner, L. F.; Cavalli, A.; Bakkers, E. Nano Lett. 2013, 13, 3802-3806.

(49) Wacaser, B. A.; Deppert, K.; Karlsson, L. S.; Samuelson, L.; Seifert, W. J. Cryst. Growth 2006, 287, 504-508.

(50) Tomioka, K.; Fukui, T. J. Phys. D: Appl. Phys. 2014, 47, 394001.

(51) Xu, H. Y.; Wang, Y.; Guo, Y. N.; Liao, Z. M.; Gao, Q.; Tan, H. H.; Jagadish, C.; Zou, J. Nano Lett. 2012, 12, 5744-5749.

(52) Boles, S. T.; Thompson, C. V.; Fitzgerald, E. A. J. Cryst. Growth 2009, 311, 1446-1450.

(53) Vogel, A. T.; de Boor, J.; Becker, M.; Wittemann, J. V.; Mensah, S. L.; Werner, P.; Schmidt, V. Nanotechnology 2011, 22, 015605.

(54) Pan, D.; Fu, M. Q.; Yu, X. Z.; Wang, X. L.; Zhu, L. J.; Nie, S. H.; Wang, S. L.; Chen, Q.; Xiong, P.; von Molnar, S.; Zhao, J. H. Nano Lett. 2014, 14, 1214-1220. 
(55) Huang, K. R.; Zhang, Z.; Zhou, Q. W.; Liu, L. W.; Zhang, X. Y.; Kang, M. Y.; Zhao, F. L.; Lu, X. B.; Gao, X. S.; Liu, J. M. Nanotechnology 2015, 26, 255706.

(56) Messing, M. E.; Hillerich, K.; Bolinsson, J.; Storm, K.; Johansson, J.; Dick, K. A.; Deppert, K. Nano Res. 2010, 3, 506-519.

(57) Persson, A. I.; Larsson, M. W.; Stenstrom, S.; Ohlsson, B. J.; Samuelson, L.; Wallenberg, L. R. Nat. Mater. 2004, 3, 677-681.

(58) Hannon, J. B.; Kodambaka, S.; Ross, F. M.; Tromp, R. M. Nature 2006, 440, 69-71.

(59) Ahtapodov, L.; Todorovic, J.; Olk, P.; Mjaland, T.; Slattnes, P.; Dheeraj, D. L.; van Helvoort, A. T.; Fimland, B. O.; Weman, H. Nano

Lett. 2012, 12, 6090-6095.

(60) Vainorius, N.; Jacobsson, D.; Lehmann, S.; Gustafsson, A.; Dick, K. A.; Samuelson, L.; Pistol, M.-E. Phys. Rev. B: Condens. Matter Mater. Phys. 2014, 89, 165423.

(61) Spirkoska, D.; Arbiol, J.; Gustafsson, A.; Conesa-Boj, S.; Glas, F.; Zardo, I.; Heigoldt, M.; Gass, M. H.; Bleloch, A. L.; Estrade, S.; Kaniber, M.; Rossler, J.; Peiro, F.; Morante, J. R.; Abstreiter, G.; Samuelson, L.; Fontcuberta i Morral, A. Phys. Rev. B: Condens. Matter Mater. Phys. 2009, 80, 245325.

(62) Furthmeier, S.; Dirnberger, F.; Hubmann, J.; Bauer, B.; Korn, T.; Schüller, C.; Zweck, J.; Reiger, E.; Bougeard, D. Appl. Phys. Lett. 2014, 105, 222109.

(63) Pandian, V.; Mohapatra, Y. N.; Kumar, V. Jpn. J. Appl. Phys. 1991, 30, 2815-2818.

(64) Blätte, M.; Schairer, W.; Willmann, F. Solid State Commun. 1970, 8, 1265-1268.

(65) Chen, J. W.; Milnes, A. G. Annu. Rev. Mater. Sci. 1980, 10, 157228.

(66) Saga, T. NPG Asia Mater. 2010, 2, 96-102.

(67) Dubrovskii, V. G.; Cirlin, G. E.; Soshnikov, I. P.; Tonkikh, A. A.; Sibirev, N. V.; Samsonenko, Y. B.; Ustinov, V. M. Phys. Rev. B: Condens. Matter Mater. Phys. 2005, 71, 205325.

(68) Plante, M. C.; LaPierre, R. R. J. Cryst. Growth 2006, 286, 394399.

(69) Tchernycheva, M.; Harmand, J. C.; Patriarche, G.; Travers, L.; Cirlin, G. E. Nanotechnology 2006, 17, 4025-4030.

(70) Li, X.; Guo, H.; Yin, Z.; Shi, T.; Wen, L.; Zhao, Z.; Liu, M.; Ma, W.; Wang, Y. J. Cryst. Growth 2011, 324, 82-87.

(71) Wagner, R. S.; Ellis, W. C. Appl. Phys. Lett. 1964, 4, 89-90.

(72) Okamoto, H. J. Phase Equilib. 1992, 13, 324-325.

(73) Rouland, J. C.; Souleau, C.; Ceolin, R. J. Therm. Anal. 1987, 32, 185-195.

(74) Seifert, W.; Borgström, M.; Deppert, K.; Dick, K. A.; Johansson, J.; Larsson, M. W.; Mårtensson, T.; Sköld, N.; Svensson, C. P. T.; Wacaser, B. A.; Wallenberg, L. R.; Samuelson, L. J. Cryst. Growth 2004, 272, 211-220.

(75) Mikkelsen, A.; Eriksson, J.; Lundgren, E.; Andersen, J. N.; Weissenrieder, J.; Seifert, W. Nanotechnology 2005, 16, 2354-2359. 